\title{
THE EXISTENCE OF ALMOST TRANSLATION INVARIANT ULTRAFILTERS ON ANY SEMIGROUP
}

\author{
TALIN PAPAZYAN
}

(Communicated by Dennis Burke)

\begin{abstract}
We present a short ultrafilter proof of a result which has applications in combinatorial number theory and which has previously relied on the theory of compact semigroups.
\end{abstract}

The existence of non-fixed ultrafilters $p$ on $N$ such that whenever $A \in p$ one has $\{x \in N: A-x \in p\} \in p$, called almost translation invariant by Galvin, has always been of interest because it is closely related to the validity of GrahamRothschild conjecture. In 1974, the conjecture was proved by Hindman [5] in ZFC. Combined with the proof in [4] which used the continuum hypothesis this yielded a $\mathrm{CH}$ proof of the existence of almost translation invariant ultrafilters. In 1975, Glazer proved their existence without using the continuum hypothesis [2]. His approach was to define an addition on ultrafilters on a semigroup $S$, so that the almost translation invariant ultrafilters become idempotents and then use Ellis' theorem (Lemma 2.9 of [3]) about the existence of idempotents in compact right topological semigroups. In this paper, we prove directly the existence of such ultrafilters on any cancellative semigroup using ultrafilter approach only.

1. Definition. Let $(S,+)$ be a semigroup, not necessarily commutative, and let $\mathscr{A}, \mathscr{B}$ be filters on $S$. For any $A \subseteq S$ we define

$$
\begin{aligned}
\Omega_{\mathscr{N}}(A) & =\{x \in S: A-x \in \mathscr{A}\}, \\
\mathscr{A}+\mathscr{B} & =\left\{A \subseteq S: \Omega_{\mathscr{B}}(A) \in \mathscr{A}\right\},
\end{aligned}
$$

where $A-x=\{y \in S: y+x \in A\}$.

The symbol + is just a notion for the above described operation on filters. The fact that $\mathscr{A}+\mathscr{B}$ is a filter follows from steps (i) and (iii) of the following lemma. (In [1], Lemma 5.15 shows that $\mathscr{A}+\mathscr{B}$ is a filter on $S$ and that + is an associative operation on the set of filters. In the following discussion the associativity of + is not needed.) All parts of the lemma are easy to prove.

Received by the editors February 7, 1989 and, in revised form, February 21, 1989.

1980 Mathematics Subject Classification (1985 Revision). Primary 54A25.

Key words and phrases. Ultrafilters. 
2. Lemma. Let $\mathscr{A}, \mathscr{B}$ be arbitrary filters on $S$.

(i) $\forall A, B \subseteq S \Omega_{\mathscr{O}}(A \cap B)=\Omega_{\mathscr{Q}} \cap \Omega_{\mathscr{A}}(B)$.

(ii) $\forall A \subseteq S \Omega_{\mathscr{A}}\left(A^{c}\right) \subseteq\left(\Omega_{\mathscr{A}}(A)\right)^{c}$; equality holds if $\mathscr{A}$ is an ultrafilter.

(iii) $\forall A, B \subseteq S, A \subseteq B \Rightarrow \Omega_{\mathscr{A}}(A) \subseteq \Omega_{\mathscr{A}}(B)$.

(iv) $\mathscr{A} \subseteq \mathscr{B} \Rightarrow \forall A \subseteq S \Omega_{\mathscr{A}}(A) \subseteq \Omega_{\mathscr{B}}(A)$.

(v) $\forall y \in S, \forall A \subseteq S \Omega_{\mathscr{G}}(A-y)=\Omega_{\mathscr{A}}(A)-y$.

(vi) $\forall A \subseteq S \Omega_{\mathscr{Q}+\mathscr{B}}(A)=\Omega_{\mathscr{O}}\left(\Omega_{\mathscr{B}}(A)\right)$.

3. Theorem. Let $(S,+)$ be any semigroup, not necessarily commutative. Then there exists an ultrafilter $p$ on $S$ such that $\forall A \in p \Omega_{p}(A) \in p$. If $S$ is cancellative then there also exists a nonfixed ultrafilter $p$ having the same property.

Proof. Zorn's Lemma gives the existence of a filter $\mathscr{F}_{\max }$ maximal with respect to the property $\forall A \in \mathscr{F}_{\max } \Omega_{\mathscr{F}_{\max }}(A) \in \mathscr{F}_{\max }$. (If $S$ is cancellative, then the filter $\mathscr{F}=\left\{A \subseteq S: A^{c}\right.$ is finite $\}$ has this property, so we may choose $\mathscr{F}_{\max }$ to refine $\mathscr{F}$; then any ultrafilter refining $\mathscr{F}_{\max }$ is nonfixed.) Our aim now is to show that $\mathscr{F}_{\max }$ is an ultrafilter. Let $p$ be an ultrafilter containing $\mathscr{F}_{\max }$. Consider $\mathscr{F}=\left\{A \subseteq S: \Omega_{p}(A) \in \mathscr{F}_{\max }\right\}$. Using Lemma 2 we can show that $\mathscr{F}$ is a filter containing $\mathscr{F}_{\max }$ and $\forall A \subseteq S$

$$
\begin{aligned}
\Omega_{\mathscr{F}}(A) & =\left\{y \in S: \Omega_{p}(A-y) \in \mathscr{F}_{\max }\right\} \\
& =\left\{y \in S: \Omega_{p}(A)-y \in \mathscr{F}_{\max }\right\}(\text { Lemma 2(v)) } \\
& =\Omega_{\mathscr{F}_{\max }}\left(\Omega_{p}(A)\right),
\end{aligned}
$$

so that $\forall A \in \mathscr{F} \Omega_{\mathscr{F}}(A) \in \mathscr{F}$ which implies that $\mathscr{F}_{\max }=\mathscr{F}$ from the maximality of $\mathscr{F}_{\max }$. Hence,

$$
\forall A \subseteq S A \in \mathscr{F}_{\max } \text { iff } \Omega_{p}(A) \in \mathscr{F}_{\max }
$$

From Lemma 2 (ii) and (1) we can easily deduce that $\forall A \in p, \quad \forall M \in \mathscr{F}_{\text {max }}$, $M \cap \Omega_{p}(A) \neq \varnothing$ so that we can consider the filter $\mathscr{F}^{\prime}$ generated by $\left\{\Omega_{p}(A) \cap M\right.$, $\left.A \in p, M \in \mathscr{F}_{\max }\right\}$. Clearly $\mathscr{F}^{\prime}$ contains $\mathscr{F}_{\max }$. Now, $\forall A \in p$ we have $\Omega_{p}(A) \in \mathscr{F}^{\prime}$, that is $A \in \mathscr{F}^{\prime}+p$ and since $p$ is an ultrafilter it follows that $\mathscr{F}^{\prime}+p=p$. Next, using Lemma 2 , we show that, for any $A \in p$ and any $M \in \mathscr{F}_{\max }, \Omega_{\mathscr{F}^{\prime}}\left(\Omega_{p}(A) \cap M\right) \in \mathscr{F}^{\prime}:$ indeed,

$$
\Omega_{\mathscr{F}^{\prime}}\left(\Omega_{p}(A) \cap M\right)=\Omega_{\mathscr{F}^{\prime}+p}(A) \cap \Omega_{\mathscr{F}^{\prime}}(M)=\Omega_{p}(A) \cap \Omega_{\mathscr{F}}(M)
$$

and

$$
\Omega_{\mathscr{F}}(M) \supseteq \Omega_{\mathscr{F}_{\max }}(M) .
$$

Thus for $B \in \mathscr{F}^{\prime}, \Omega_{\mathscr{F}^{\prime}}(B) \in \mathscr{F}^{\prime}$ and the maximality of $\mathscr{F}_{\max }$ gives $\mathscr{F}^{\prime}=\mathscr{F}_{\max }$. So, for all $A \in p, \Omega_{p}(A) \in \mathscr{F}_{\max }$, which implies that $\mathscr{F}_{\max }=p$ by (1). Thus $\mathscr{F}_{\max }$ is an ultrafilter. 
In conclusion, we should point out that the structure of the above proof is basically the same as that of Ellis' algebraic proof though the details differ.

\section{ACKNOWLEDGMENT}

I would like to express my gratitude to Professor J. S. Pym for his valuable advice and suggestions.

\section{REFERENCES}

1. J. F. Berglund and N. Hindman, Filters and the weak almost periodic compactification of a discrete semigroup, Trans. Amer. Math. Soc. 284 (1984), 1-38.

2. W. W. Comfort, Ultrafilters: some old and some new results, Bull. Amer. Math. Soc. 83 (1977), 417-455.

3. R. Ellis, Lectures on topological dynamics, Benjamin, New York, 1969.

4. N. Hindman, The existence of certain ultrafilters on $N$ and a conjecture of Graham and Rothschild, Proc. Amer. Math. Soc. 36 (1972), 341-346.

5. _ Finite sums from sequences within cells of a partition of $N, \mathrm{~J}$. Comb. Theory (Ser. A) 17 (1974), 1-11.

Department of Pure Mathematics, Hicks Building, University of Sheffield, Sheffield S3 7RH 